\title{
First evidence for molecular interfaces between outflows and ambient cloud in high-mass star forming regions?
}

\author{
C. Codella \\ INAF - Istituto di Radioastronomia, Sezione di Firenze, Largo E. Fermi 5, 50137 Firenze, Italy \\ codella@arcetri.astro.it \\ S. Viti and D.A. Williams \\ Department of Physics and Astronomy, University College London, Gower Street, London, WC1E6BT \\ sv@star.ucl.ac.uk, daw@star.ucl.ac.uk \\ and \\ R. Bachiller ${ }^{3}$ \\ Observatorio Astronómico Nacional (IGN), Alfonso XII 3, E-28014 Madrid, Spain \\ bachiller@oan.es
}

\begin{abstract}
We present new observations of the CepA-East region of massive star formation and describe an extended and dynamically distinct feature not previously recognised. This feature is present in emission from $\mathrm{H}_{2} \mathrm{CS}$, OCS, $\mathrm{CH}_{3} \mathrm{OH}$, and $\mathrm{HDO}$ at $-5.5 \mathrm{~km} \mathrm{~s}^{-1}$, but is not traced by conventional tracers of star forming regions $\mathrm{H}_{2} \mathrm{~S}, \mathrm{SO}_{2}, \mathrm{SO}, \mathrm{CS}$. The feature is extended up to at least $0.1 \mathrm{pc}$. We show that the feature is neither a hot core nor a shocked outflow. However, the chemistry of the feature is consistent with predictions of a model of an eroding interface between a fast wind and a dense core; mixing between the two media occurs in the interface on a timescale of 10-50 years. If these observations are confirmed by detailed maps and by detections in species also predicted to be abundant (e.g. $\mathrm{HCO}^{+}, \mathrm{H}_{2} \mathrm{CO}$, and $\mathrm{NH}_{3}$ ) this feature would be the first detection of such an interface in regions of massive star formation. An important implication of the model is that a significant reservoir of sulfur in grain mantles is required to be in the form of OCS.
\end{abstract}

Subject headings: ISM: individual (CepA-East) — ISM: molecules — radio lines: ISM — star formation

\section{Introduction}

To explore the interactions of young stellar objects (YSOs) with their environments, we recently carried out mm-wavelength molecular line observations towards star forming regions (SFRs) with well defined and bright high-velocity components. In particular, we mapped the well known Cepheus A (CepA; Garay et al. 1996, and references therein) SFR in several shock-chemistry tracers such as $\mathrm{H}_{2} \mathrm{~S}, \mathrm{SO}_{2}$, and $\mathrm{HDO}$ (Codella et al. 2003, 2005). Those results show that the group of B-type stars located in CepA-East producing a hot core (Martín-Pintado et al. 2005), are also associated with multiple mass loss processes. In particular, beside the already known three flows pointing in the SW, NE, and SE directions, a fourth outflow flowing towards the South has been detected thanks to the shock-chemistry tracers. CepA-East can thus be considered an ideal laboratory in which to study how outflow motions affect the gas, from both the kinematical and chemical points of view. Codella et al. (2005) have already presented a multi-species and multi- 
line mm-survey of the central region of CepA-East where the YSOs are located. Using the 30-m IRAM antenna, the authors detected emission in different transitions of 21 molecular species tracing a wide range of physical conditions. Analysis of these spectra shows that different molecules exhibit different spectral behaviours and that three classes can be distinguished: (i) hot core molecules (e.g. $\mathrm{HC}^{18} \mathrm{O}^{+}, \mathrm{CH}_{3} \mathrm{C}_{2} \mathrm{H}$ ) emitting only at the velocity of the hot core $\left(-10.7 \mathrm{~km} \mathrm{~s}^{-1}\right)$ and with no line wings, (ii) outflow molecules (e.g. CS, SiO, $\mathrm{H}_{2} \mathrm{~S}, \mathrm{SO}_{2}$, and $\mathrm{SO}$ ) spanning the whole range of observed outflowing velocities so that bright wings are added to the hot core emission, and (iii) four species (OCS, $\mathrm{H}_{2} \mathrm{CS}$, HDO, and $\mathrm{CH}_{3} \mathrm{OH}$ ) which are associated with wings and which, in addition, clearly show a redshifted spectral peak at -5.5 $\mathrm{km} \mathrm{s}^{-1}$, well separated from the hot core peak. While the peak at $-10.7 \mathrm{~km} \mathrm{~s}^{-1}$ is tracing the high-density material hosting the YSOs and the wings are tracing the multiple outflows, the origin of the redshifted spectral peak is unknown. The Codella et al. (2005) data did not allow us to clarify the spatial distribution of this spectral peak and to establish if it is tracing a small structure or it is related with an extended component. It is worth noting that, as far as we know, this is the first study to reveal duality in the line-wing profiles observed in outflows driven by YSOs, i.e that OCS, $\mathrm{H}_{2} \mathrm{CS}$, HDO, and $\mathrm{CH}_{3} \mathrm{OH}$ (hereafter called double-peaked species) have a different behaviour with respect to $\mathrm{CS}, \mathrm{SiO}, \mathrm{H}_{2} \mathrm{~S}, \mathrm{SO}_{2}$, and $\mathrm{SO}$ (hereafter called single-peaked species). This suggests that the redshifted spectral peak could be tracing a different gas component with respect to the gas outflowing at the other velocities as well as to the hot core gas, and indicates that high angular resolution observations are required for a detailed analysis.

In this Letter we present observations which clarify the spatial distribution of the redshifted spectral peak at $-5.5 \mathrm{~km} \mathrm{~s}^{-1}$. In addition, we produce chemical models suggesting that we are probably observing the first direct evidence of turbulent interfaces (i) where the outflow gas impinges on and detach dense gas, and (ii) in which dynamical mixing and diffusion are occurring between the mass ejected from a newly-formed massive YSO and the interstellar cloud from which it was formed. In the following, the kinematical component at $-5.5 \mathrm{~km} \mathrm{~s}^{-1}$ will be referred to as the I-feature.

\section{The spatial distribution of the I-feature}

The main goal of the observations was to determine the spatial distribution of the I-feature observed towards CepA-East. In order to select the best molecular tracers, following Codella et al. (2005), we noted that a good compromise between high angular resolution, a simple spectral pattern, and an intense line emission was represented by $\mathrm{H}_{2} \mathrm{CS}$, and in particular by its $6_{16}-5_{15}$ transition at $202923.55 \mathrm{MHz}\left(E_{\mathrm{u}}=47 \mathrm{~K}\right)$. Thus, we mapped a region of $\sim 1^{\prime} \times 2^{\prime}$ in $\mathrm{H}_{2} \mathrm{CS}\left(6_{16}-5_{15}\right)$ on 2004 June with the 30-m IRAM radiotelescope of Pico Veleta (Spain). We used a sampling of $10^{\prime \prime}$ around the coordinates of HW2 YSO, which is thought to be among the main drivers of the CepA-East outflows and it represents the center of the present map, whereas a spacing of $20^{\prime \prime}$ was chosen to scan coarsely the outer part of the cloud. The system temperature, $T_{\text {sys }}$, was $\sim 500 \mathrm{~K}$, the HPBW was $12^{\prime \prime}$, while the pointing was accurate to within $2^{\prime \prime}-3^{\prime \prime}$. As spectrometer, we used an autocorrelator with a configuration providing a velocity resolution of $0.06 \mathrm{~km} \mathrm{~s}^{-1}$, successively smoothed to $0.92 \mathrm{~km} \mathrm{~s}^{-1}$. The spectra were calibrated with the standard chopper wheel method and reported here in units of main-beam brightness temperature $\left(T_{\mathrm{MB}}\right)$ : the average r.m.s. is $\sim 20 \mathrm{mK}$.

Figure $1 a$ reports the velocity channel maps of the $\mathrm{H}_{2} \mathrm{CS}\left(6_{16}-5_{15}\right)$ emission. Each panel shows the emission integrated over a velocity interval of $2 \mathrm{~km} \mathrm{~s}^{-1}$; the ambient velocity $\left(V_{\mathrm{LSR}}\right)$ is -10.7 $\mathrm{km} \mathrm{s}^{-1}$ (e.g. Codella et al. 2005). In summary, $\mathrm{H}_{2} \mathrm{CS}\left(6_{16}-5_{15}\right)$ emission is associated (i) with the central position, where the YSO cluster is located and where a hot core has been detected (MartínPintado et al. 2005), and (ii) with the four outflow directions, NE, SE, S, and SW, confirming $\mathrm{H}_{2} \mathrm{CS}$ as a tracer of high-temperature and/or shocked regions. In particular, the new $\mathrm{H}_{2} \mathrm{CS}$ maps confirm that the spatial distribution of the I-feature is not limited to the central position tracing also the SW and southern outflows, as clearly shown by the -5.5 and $-3.5 \mathrm{~km} \mathrm{~s}^{-1}$ panels in Fig. 1a.

Examples of $\mathrm{H}_{2} \mathrm{CS}\left(6_{16}-5_{15}\right)$ line profiles are shown in Fig. $1 b$ which compares the spectra observed at the $\left(0^{\prime \prime},+10^{\prime \prime}\right)$ and $\left(0^{\prime \prime},-10^{\prime \prime}\right)$ offsets with 
that observed at the $\left(0^{\prime \prime}, 0^{\prime \prime}\right)$ position (Codella et al. 2005). Given the HPBW, the three spectra are sampling different regions of CepA-East. The I-feature is still present in the southern position and it is even more redshifted, approaching the $-5 \mathrm{~km} \mathrm{~s}^{-1}$ velocity, thus suggesting the presence of a velocity gradient. In order to make the same comparison with another tracer of the I-feature, we observed the $\mathrm{CH}_{3} \mathrm{OH}\left(5_{\mathrm{K}}-4_{\mathrm{K}}\right)$ transition at $241.8 \mathrm{GHz}$ towards the $\left(0^{\prime \prime},+10^{\prime \prime}\right)$ and $\left(0^{\prime \prime},-10^{\prime \prime}\right)$ positions. In this case, $T_{\text {sys }} \simeq 650 \mathrm{~K}$, the HPBW $=10^{\prime \prime}$, and the resulting spectra were smoothed to $0.39 \mathrm{~km} \mathrm{~s}^{-1}$, with a r.m.s. $\sim 80 \mathrm{mK}$. Fig. $1 c$ compares the profiles due to the high excitation $\left(E_{\mathrm{u}}=115 \mathrm{~K}\right) \mathrm{CH}_{3} \mathrm{OH}\left(5_{4}-4_{4} \mathrm{~A}^{ \pm}\right)$transition at $241806.51 \mathrm{MHz}$, which according to the $\left(0^{\prime \prime}, 0^{\prime \prime}\right)$ spectrum, can be considered as the best tracer of the I-feature among the lines of the $\mathrm{CH}_{3} \mathrm{OH}\left(5_{\mathrm{K}^{-}}\right.$ $\left.4_{\mathrm{K}}\right)$ pattern. Also in this case, the I-feature is still present in the southern position and again it seems more redshifted, in agreement with the $\mathrm{H}_{2} \mathrm{CS}$ data. It is worth noting that the two continuous lines across the methanol $\left(0^{\prime \prime}, 0^{\prime \prime}\right)$ spectrum refer to the two velocity components of the $\mathrm{CH}_{3} \mathrm{OH}\left(5_{-4}-4_{-4}\right.$ E) transition $\left(E_{\mathrm{u}}=122 \mathrm{~K}\right)$ : the $-5.5 \mathrm{~km} \mathrm{~s}^{-1}\left(5_{-4^{-}}\right.$ $\left.4_{-4} \mathrm{E}\right)$ emission is blended with the $-10.7 \mathrm{~km} \mathrm{~s}^{-1}$ $\left(5_{4}-4_{4} \mathrm{~A}^{ \pm}\right)$feature, producing a broad line. Finally, Fig. $1 d$ reports the $\operatorname{HDO}\left(1_{10}-1_{11}\right)\left(E_{\mathrm{u}}=47\right.$ $\mathrm{K})$ profiles that were also reported in the Codella et al. (2005) paper. Since the angular resolution is definitely higher $\left(\mathrm{HPBW}=31^{\prime \prime}\right)$ we show the spectra taken in the $\left(0^{\prime \prime},+20^{\prime \prime}\right)$ and $\left(0^{\prime \prime},-20^{\prime \prime}\right)$ positions to sample different parts of CepA-East. The HDO data are well in agreement with the picture given above, showing how bright is the I-feature as observed towards the southern position.

We conclude that there is a previously undetected structure extending toward the South from the central position giving rise to emission at $5.5 \mathrm{~km} \mathrm{~s}^{-1}$ in lines of $\mathrm{H}_{2} \mathrm{CS}$, OCS, HDO, and $\mathrm{CH}_{3} \mathrm{OH}$. The structure appears to be at least 0.1 $\mathrm{pc}$ in length.

\section{The origin of the I-feature}

In this Section we consider possible origins for the extended I-feature at $-5.5 \mathrm{~km} \mathrm{~s}^{-1}$. Since CepA-East is a YSO cluster the possibility that this emission comes from a second hot core seems, at first sight, likely. However, this cannot be the case because: (i) the emission is extended and not compact as it would be if it came from a hot core (see Fig. 1). In addition, (ii) Figure 2 shows that what makes the I-feature peculiar is that some line ratios such as OCS/SO, $\mathrm{H}_{2} \mathrm{CS} / \mathrm{SO}_{2}$ or $\mathrm{CH}_{3} \mathrm{OH} / \mathrm{H}_{2} \mathrm{~S}$ are definitely much higher than those measured towards the $-10.7 \mathrm{~km} \mathrm{~s}^{-1}$ component. In other words, although we cannot exlude that conventional hot core tracers, including, SO, $\mathrm{SO}_{2}$, and $\mathrm{H}_{2} \mathrm{~S}$, can have some weak emission at $5.5 \mathrm{~km} \mathrm{~s}^{-1}$, they are not tracing the I-feature. Finally, (iii) we find that the column densities of the double peaked species in the I-feature $\left(\mathrm{CH}_{3} \mathrm{OH}\right.$ : $310^{15} \mathrm{~cm}^{-2}$; OCS: $510^{13} ; \mathrm{H}_{2} \mathrm{CS}$ : $10^{14}$; HDO: $610^{14}$; Codella et al. 2005), calculated assuming extended emission, are lower that those typically found in hot cores (Jacq et al. 1990; Millar \& Hatchell 1998). We conclude that there are both morphological and chemical reasons for excluding a hot core as the origin of the I-feature.

We have also investigated the possibility that the I-feature is simply the emission from a shocked molecular outflow. This possibility has been proposed in the Codella et al. (2005) paper, where the observations were compared with the theoretical calculations recently reported by Wakelam et al. (2004). However, a closer inspection of such model suggests that the theoretical abundances of the single-peaked species are not reproduced and that both single and double peaked species abundances, once converted into column densities (i.e. taking into consideration the geometry) are more typical of hot cores densities and hence too high. In addition, it is difficult to understand why standard shock tracers like $\mathrm{SiO} . \mathrm{SO}_{2}$, and $\mathrm{H}_{2} \mathrm{~S}$ do not show the same profile observed with the doublepeaked species, as suggested by shock-chemistry models and as happens in the well-studied chemical rich outflows BHR71 and L1157 (Garay et al. 1998; Bachiller \& Peréz Gutiérrez 1999).

A new explanation is therefore required. We now investigate the possibility that the I-feature arises from a molecular interface between the outflow gas and the ambient medium. Highly turbulent interfaces should be present in all environments where jets or outflows interact with the surrounding molecular clouds. Such interfaces have been shown to be characterized by a chemistry which is quite distinct in nature from that of typical dense cores and would not be achieved by 
any modification of conventional cold cloud chemistry. All chemical interface models, whether with turbulent mixing or diffusion, explore the consequences of mixing warm largely ionized gas with cold dense and mainly neutral gas (e.g. Lim, Rawlings, \& Williams 2001; Rawlings \& Hartquist 1997). There is as yet no direct evidence of the existence of such interfaces in high-mass SFRs, while in low-mass SFRs, observations of enhanced $\mathrm{HCO}^{+}$towards several Class 0 objects can be interpreted as coming from the walls of the outflow cavity (Hogerheijde et al. 1998; Rawlings, Taylor, \& Williams 2000; Redman et al. 2004). In order to test this hypothesis we make use of the Viti et al. (2002) interface model and attempt to reproduce the different trends displayed by the single-peaked and double-peaked species. Note that since the model does not include the deuterium chemistry, we could not calculate the abundance of HDO.

The main physical characteristics often adopted for an interface are: low visual extinctions $(\leq 1.5$ mags); high gas densities $\left(n_{\mathrm{H}_{2}} \simeq 10^{4}-10^{7} \mathrm{~cm}^{-3}\right)$, high radiation fields compared to the mean interstellar radiation field (here called one Habing), short lifetimes $(\leq 100 \mathrm{yr})$ and relatively high temperatures $\left(T_{\text {kin }} \geq 100 \mathrm{~K}\right)$ due to non-dissociative shocks associated with the highly turbulent interface between the outflow and the molecular core. Note that although timescales may be very short, the continual erosion of dense material by the wind or jet re-supplies the interface, so a near steadystate pertains. The temperature and density of the region, as deduced from the molecular observations (Codella et al. 2005), are $\sim 200 \mathrm{~K}$ and $10^{6}-10^{7} \mathrm{~cm}^{-3}$, respectively. We have therefore run a small grid of models using these values and varying the visual extinction (0.5-1.5 mags) and the radiation fields (5-100 Habing). A further parameter that we investigate is the form that sulfur takes once depleted on the grains, before the mantles are evaporated. We have considered $100 \%$ in solid $\mathrm{H}_{2} \mathrm{~S}, 100 \%$ in solid OCS and several mixtures in between. Note that while it is often assumed that most simple species, once frozen out, react with hydrogen atoms to form saturated species, recently doubts have been raised regarding the form that sulfur takes once it depletes onto the grains. In particular, the detection of solid OCS (Palumbo, Geballe, \& Tielens 1997) and the non- detection of $\mathrm{H}_{2} \mathrm{~S}$ ices (van Dishoeck \& Blake 1998) have raised the possibility that OCS may be the main reservoir of sulfur on the grains. It is not unrealistic to assume that before evaporation most of the sulfur is in the form of OCS: studies of the effects of UV on grains (e.g. Mannella, Palumbo, \& Baratta 1997) have shown that species such as $\mathrm{H}_{2} \mathrm{O}$ for example, are easily dissociated by radiation; once the oxygen is freed, it is believed to react with $\mathrm{CO}$, which is the third most abundant species on the grains, to form $\mathrm{CO}_{2}$. It is not unreasonable to assume that a similar process occurs with $\mathrm{H}_{2}$ S: i.e. that once atomic sulfur is freed it reacts efficiently with $\mathrm{CO}$ to form OCS.

The aim of this exercise is not to match perfectly the observed column densities but to see whether there is an epoch when a molecular outflow-ambient interface could give rise to high abundances of $\mathrm{CH}_{3} \mathrm{OH}$, OCS and $\mathrm{H}_{2} \mathrm{CS}$ while $\mathrm{SO}$, $\mathrm{SO}_{2}$ and $\mathrm{H}_{2} \mathrm{~S}$ remained low. Figure 3 shows one of our best fit models, where, in general, the behaviour is as expected: the double-peaked species show a clear anti-correlation with respect to the single-peaked family. Note that in any case the molecular abundances of all the species ultimately ( $\geq 100 \mathrm{yr}$ ) decline as the radiation field induces photodissociation, but, as mentioned earlier, the interface is turbulent and therefore a continuous erosion of material will constantly replenish the interface gas. A radiation field larger than 10 Habing seems to destroy most of the doublypeaked species too early. A density lower than $10^{6}$ $\mathrm{cm}^{-3}$ does not produce enough methanol. But the most interesting constraint is the amount of sulfur (at least 90\%) that needs to be in solid OCS before evaporation occurs. Thus, these calculations suggest that the observations are consistent with (but do not prove) a model of an outflow-ambient turbulent interface with the following physical conditions: $n_{\mathrm{H}_{2}} \simeq 10^{6}-10^{7} \mathrm{~cm}^{-3}, T_{\text {kin }} \sim 200 \mathrm{~K}$, and a radiation field between 5 and 10 Habing. According to this modelling, our observational data may be evidence of the presence of a molecular interface in high mass star forming regions. As well as indicating high abundance of the double-peaked species, our model:

1. requires high abundances of solid OCS, and an entrainment of sufficient material into the interface on timescales of 10-50 years. While 
it is feasible that OCS on ices is abundant in an environment where the UV radiation field is strong, it is clear that experimental as well as observational studies on ices are desirable to confirm such picture;

2. predicts high abundances of other species, including $\mathrm{HCO}^{+}, \mathrm{H}_{2} \mathrm{CO}$, and $\mathrm{NH}_{3}$. To confirm the presence of a molecular interface between outflow and ambient gas, we thus suggest further observations to map CepA-East in these species as well as OCS lines.

SV acknowledges financial support from an individual PPARC advanced fellowship.

\section{REFERENCES}

Bachiller, R., \& Peréz Gutiérrez, M. 1999, ApJ, 487, L93

Codella, C., Bachiller, R., Benedettini, M., \& Caselli, P. 2003, MNRAS, 341, 707

Codella, C., Bachiller, R., Benedettini, M., Caselli, P., Viti, S., \& Wakelam, V. 2005, MNRAS, 361, 244

Garay, G., Ramírez, S., Rodríguez, L.F., Curiel, S., \& Torrelles, J.M. 1996, ApJ, 459, 193

Garay, G., Köhnenkamp, I., Bourke, T.L., Rodríguez, \& Lehtinen, K.K. 1998, ApJ, 509, 768

Hogerheijde, M.R., van Dishoeck, E.F., Blake, G.A., \& van Langevelde, H.J. 1998, ApJ, 502, 315

Jacq, T., Walmsley, C.M., Henkel, C., Baudry, A., Mauersberger, R., \& Jewell, P.R. 1990, A\&A, 228,447

Lim, A.J., Rawlings, J.M.C., \& Williams, D.A. 2001, A\&A, 376, 336

Mannella, V., Palumbo, M.E., \& Baratta, G.A. 2004, ApJ, 615, 1073

Martín-Pintado, J., Jiménez-Serra, I., RodríguezFranco, A., Martín, S., \& Thum, C. 2005, ApJ, 628, L61
Millar, T.J., \& Hatchell, J., 1998, in Chemistry and Physics of Molecules and Grains in Space, Faraday Discussions N. 109, The Faraday Division of the Royal Society of Chemistry, London, 15

Palumbo, M.E., Geballe, T.R., \& Tielens, A.G.G.M. 1997, ApJ, 479, 839

Rawlings, J.M.C., \& Hartquist, T.W. 1997, ApJ, 487,672

Rawlings, J.M.C., Taylor, S.D., \& Williams, D.A. 2000, MNRAS, 313, 461

Redman, M.P., Rawlings, J.M.C., Yates, J.A., \& Williams, D.A. 2004, MNRAS, 352, 243

van Dishoeck, E.F., \& Blake, G.A. 1998, ARA\&A, 36,317

Viti, S., Natarajan, S., \& Williams, D.A. 2002, MNRAS, 336, 797

Wakelam, V., Caselli, P., Herbst, E., Ceccarelli, C., \& Castets, A. 2004, A\&A, 422, 159

This 2-column preprint was prepared with the AAS IATEX macros v5.2. 

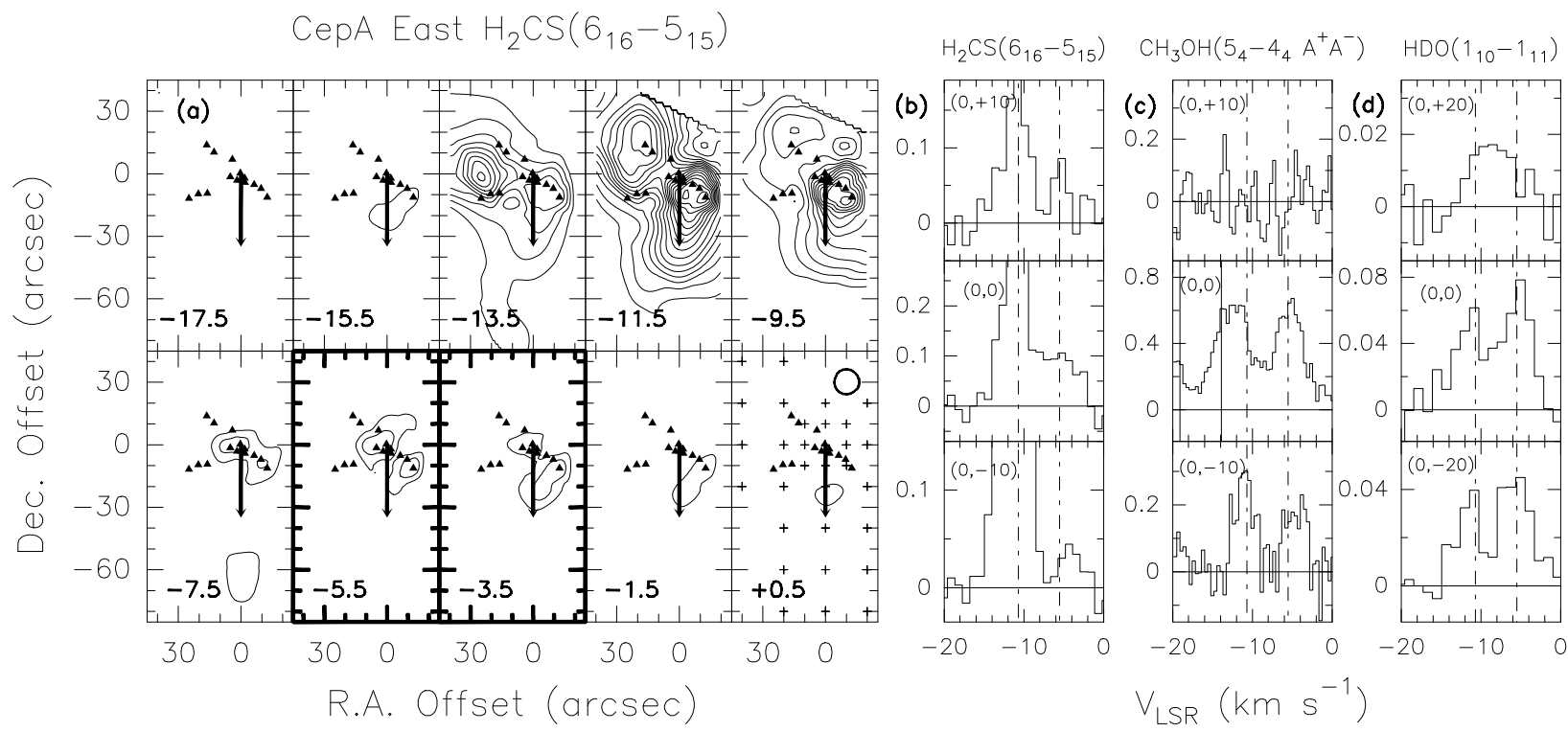

Fig. 1.- (a) Channel map of the $\mathrm{H}_{2} \mathrm{CS}\left(6_{16}-5_{15}\right)$ emission towards CepA East. Each panel shows the emission integrated over a velocity interval of $2 \mathrm{~km} \mathrm{~s}^{-1}$ centred at the value given in the bottom left corner. The thick boxes point out the main velocities of the I-feature (see text). The position offsets are relative to $\alpha_{2000}$ $=22^{\mathrm{h}} 56^{\mathrm{m}} 17^{\mathrm{s}} .9, \delta_{2000}=+62^{\circ} 01^{\prime} 49^{\prime \prime} .7$, which are the HW2 coordinates. The empty circle shows the IRAM 30-m beam (HPBW; 12" $2^{\prime \prime}$, while the small crosses mark the observed positions. The triangles stand for the VLA $2 \mathrm{~cm}$ continuum components which trace two strings of sources arising in shocks (Garay et al. 1996). The black arrow points out the direction of the 0.6 pc collimated $\mathrm{H}_{2} \mathrm{~S}$ outflow discovered by Codella et al. (2003). The contours range from 80 to $1120 \mathrm{mK} \mathrm{km} \mathrm{s}^{-1}$. The first contour and the step correspond to about $4 \sigma$ (where $\sigma$ is the r.m.s. of the map). (b) and $(c): \mathrm{H}_{2} \mathrm{CS}\left(6_{16}-5_{15}\right)\left(E_{\mathrm{u}}=47 \mathrm{~K}\right)$ and $\mathrm{CH}_{3} \mathrm{OH}\left(5_{4}-4_{4} \mathrm{~A}^{ \pm}\right)$ $\left(E_{\mathrm{u}}=115 \mathrm{~K}\right)$ spectra in $T_{\mathrm{MB}}$ scale observed at the $\left(0^{\prime \prime},+10^{\prime \prime}\right)$ and $\left(0^{\prime \prime},-10^{\prime \prime}\right)$ offsets and compared with those observed at the $\left(0^{\prime \prime}, 0^{\prime \prime}\right)$ position (Codella et al. 2005). The HPBWs of the $\mathrm{CH}_{3} \mathrm{OH}$ and $\mathrm{H}_{2} \mathrm{CS}$ observations are $10^{\prime \prime}$ and $12^{\prime \prime}$, respectively. The $\mathrm{H}_{2} \mathrm{CS}$ spectra have been truncated to stress the I-feature, pointed out by a dashed line as well as the ambient velocity, $-10.7 \mathrm{~km} \mathrm{~s}^{-1}$. The two continuous line in the $\left(0^{\prime \prime}, 0^{\prime \prime}\right) \mathrm{CH}_{3} \mathrm{OH}$ panel refer to the two velocity components of the $\left(5_{-4} 4_{-4} \mathrm{E}\right)$ transition $\left(E_{\mathrm{u}}=122 \mathrm{~K}\right)$. $(d)$ : Same as $(\mathrm{b})$ and (c) for the $\operatorname{HDO}\left(1_{10}-1_{11}\right)\left(E_{\mathrm{u}}=47 \mathrm{~K}\right)$ emission (Codella et al. 2005). Since in this case the HPBW is $31^{\prime \prime}$, the spectra taken at the $\left(0^{\prime \prime},+20^{\prime \prime}\right)$ and $\left(0^{\prime \prime},-20^{\prime \prime}\right)$ are shown. 


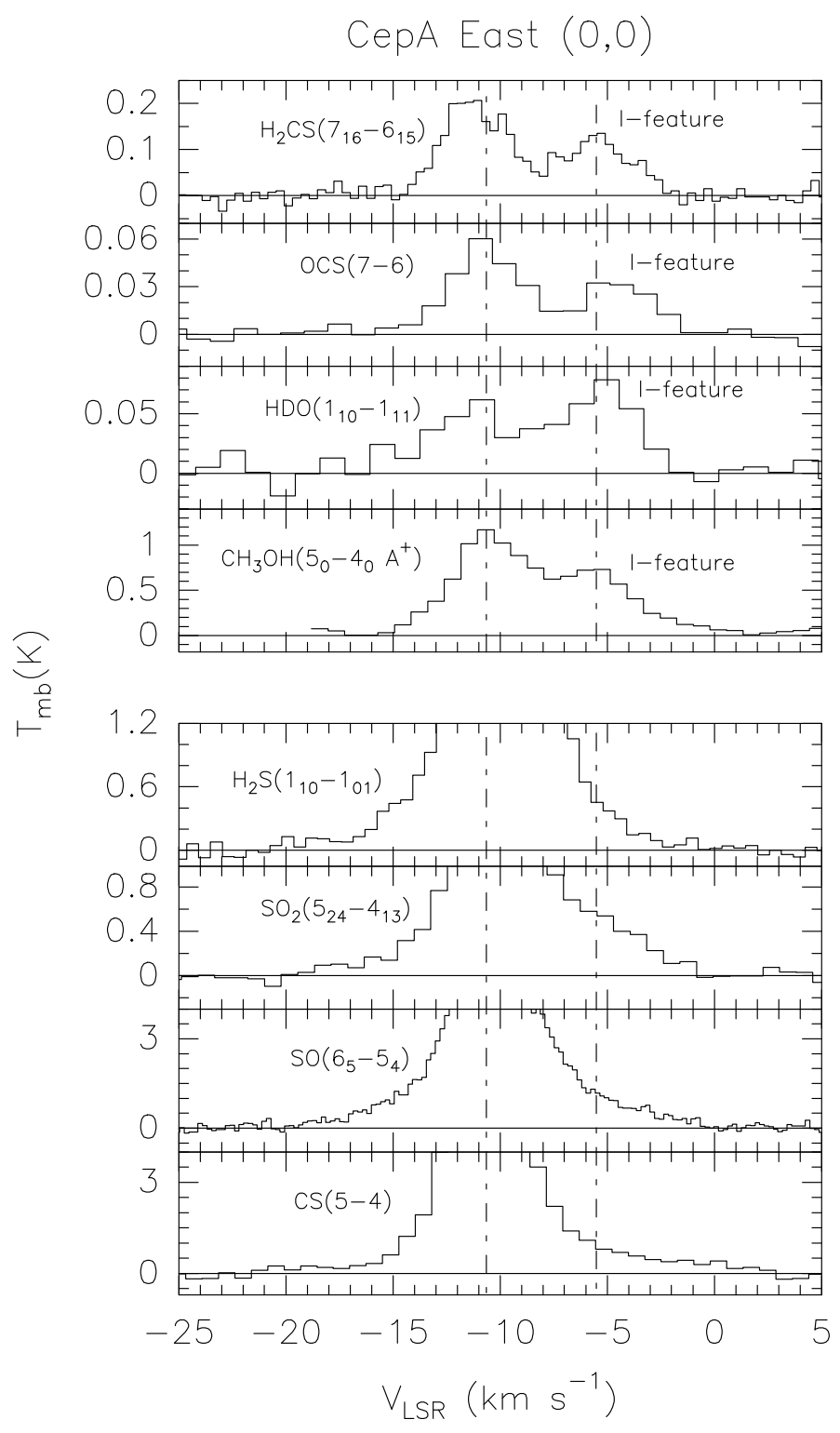

Fig. 2.- Molecular line profiles observed towards CepA East (Codella et al. 2005): species and transitions are reported. Two different kinds of line profiles are observed: (i) $\mathrm{H}_{2} \mathrm{~S}, \mathrm{SO}_{2}$, SO, and CS (lower panels) are associated with extended wings spanning the whole range of outflow velocities, whereas (ii) $\mathrm{H}_{2} \mathrm{CS}$, OCS, $\mathrm{HDO}$, and $\mathrm{CH}_{3} \mathrm{OH}$ (upper panels) are associated with wings and in addition show a redshifted secondary peak at $-5.5 \mathrm{~km} \mathrm{~s}^{-1}$ (called I-feature in the text), well separated from the ambient velocity $\left(-10.7 \mathrm{~km} \mathrm{~s}^{-1}\right.$ ). The dashed lines stand for these two velocities. The spectra in the lower panels have been truncated in order to focus the attention on the spectral wings and to stress the absence of the secondary peak clearly observed thanks to the $\mathrm{H}_{2} \mathrm{CS}$, OCS, $\mathrm{HDO}$, and $\mathrm{CH}_{3} \mathrm{OH}$ emissions. 


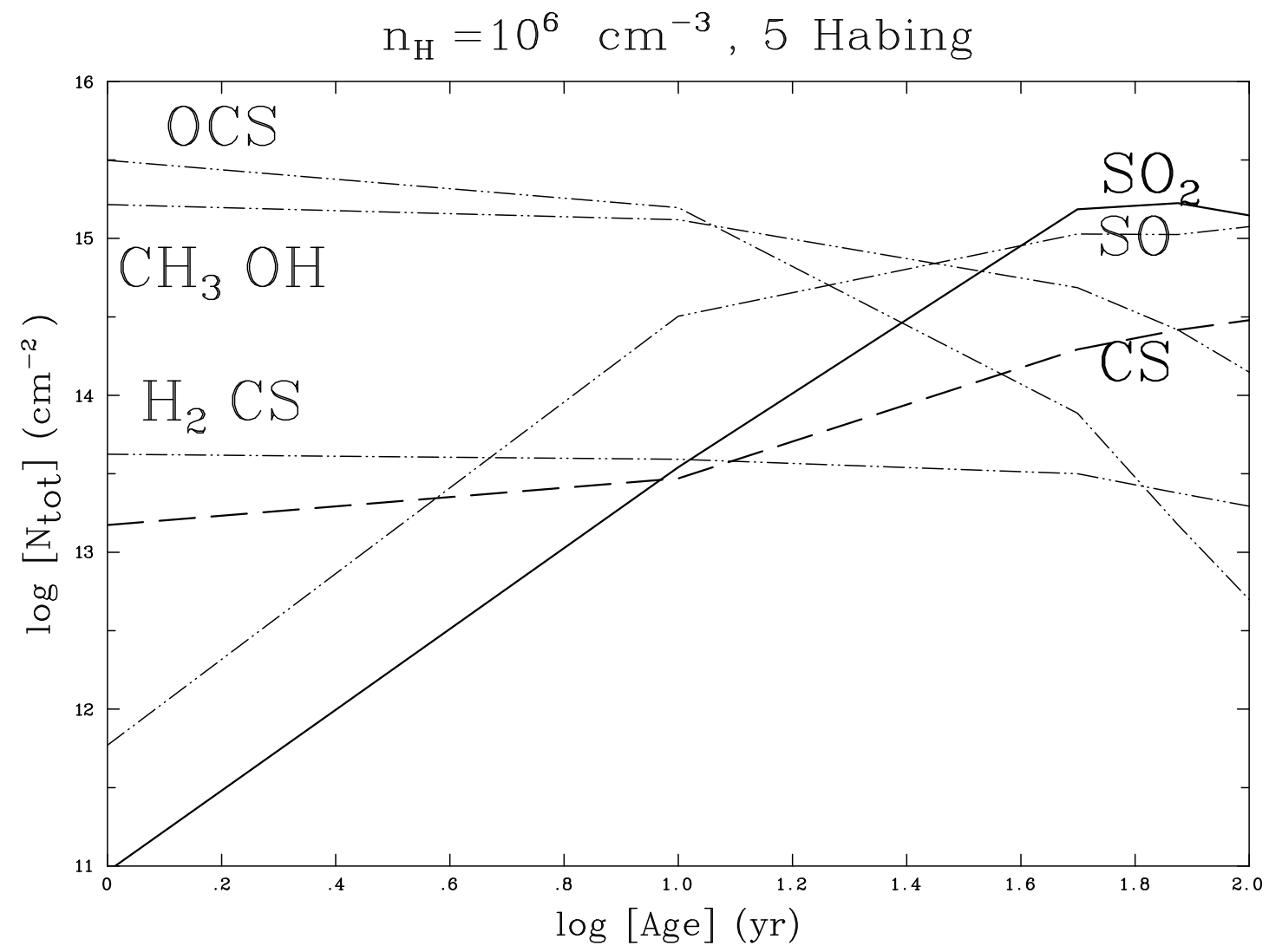

Fig. 3.- Column densities of the double-peaked $\left(\mathrm{H}_{2} \mathrm{CS}, \mathrm{OCS}, \mathrm{CH}_{3} \mathrm{OH}\right)$ and the single-peaked species $\left(\mathrm{H}_{2} \mathrm{~S}\right.$, $\mathrm{SO}_{2}, \mathrm{SO}, \mathrm{CS}$ ) as a function of time for one of the best fit models of molecular interfaces (see Sect. 3): the gas density is $10^{6} \mathrm{~cm}^{-3}$, while the radiation field is 5 Habing. Note that the column density of $\mathrm{H}_{2} \mathrm{~S}$ is less than $10^{11} \mathrm{~cm}^{-2}$ and hence it is undetectable. Before evaporation, $100 \%$ of the sulfur on the grains is in the form of OCS. 